\title{
GUTIÉRREZ GÓMEZ, EDGAR [2012]. EL ABSURDO DE LA VIDA. AYACUCHO [PERÚ]: COPYGRAPH BAUTISTA, 129 PP.
}

\author{
GUTIÉRREZ GÓMEZ, EDGAR (2012). THE ABSURDITY OF LIFE. \\ AYACUCHO (PERU): COPYIST BAUTISTA, 129 PP.
}

(iD) Manuel Abraham Paz y Miño Conde ${ }^{1^{*}}$

mpaz@ulima.edu.pe

${ }^{1}$ Universidad de Lima, Lima, Perú

*Correspondencia: Manuel Abraham Paz y Miño Conde. Email: mpaz@ulima.edu.pe

Recibido: 19.04.19 | Aprobado: 20.06.19

\section{RESUMEN}

Como toda expresión humana, el sentido de la vida se puede abordar de modo optimista o pesimista. Sobre el primer modo, tenemos los libros de: a) Juan A. Mackay, filósofo y teólogo presbiteriano escocés, El sentido de la vida y otros ensayos de 1978 (3ra. ed.), donde habla en realidad del sentido de la hombridad, la vocación, la verdad, la amistad, el universo y lo cristiano; el autobiográfico de Moisés Chávez Ramos, escritor y hebraísta evangélico, Filosofía de la vida (s/f) donde concibe a esta "como la búsqueda del propósito y del sentido de la vida” (p. 7). b) Ángel Peña Benito, sacerdote agustino, Vale la pena vivir de 2006, quien afirma que "(e)l sentido de la vida está en Dios, que nos ama, aunque, a veces, esté oculto y silencioso" (p. 4).

Palabras clave: El absurdo de la vida, Ayacucho, copygraph Bautista

\begin{abstract}
Like all human expression, the meaning of life can be approached in an optimistic or pessimistic way. On the first way, we have the books of: a) John A. Mackay, Scottish Presbyterian philosopher and theologian, The Meaning of Life and Other Essays of 1978 (3rd ed.), where he actually talks about the meaning of manhood, vocation, truth, friendship, the universe and the Christian; the autobiography of Moisés Chávez Ramos, writer and evangelical Hebraist, Filosofía de la vida (s/f) where he conceives of life "as the search for the purpose and meaning of life" (p. 7). b) Ángel Peña Benito, Augustinian priest, Worth Living in 2006, who affirms that "(e)l meaning of life is in God, who loves us, although, at times, he is hidden and silent" (p. 4).
\end{abstract}

Keywords: The absurdity of life, Ayacucho, Bautista copygraph 
Como toda expresión humana, el sentido de la vida se puede abordar de modo optimista o pesimista. Sobre el primer modo, tenemos los libros de:

a) Juan A. Mackay, filósofo y teólogo presbiteriano escocés, El sentido de la vida y otros ensayos de 1978 (3ra. ed.), donde habla en realidad del sentido de la hombridad, la vocación, la verdad, la amistad, el universo y lo cristiano; el autobiográfico de Moisés Chávez Ramos, escritor y hebraísta evangélico, Filosofía de la vida (s/f) donde concibe a esta "como la búsqueda del propósito y del sentido de la vida" (p. 7). b) Ángel Peña Benito, sacerdote agustino, Vale la pena vivir de 2006, quien afirma que "(e)l sentido de la vida está en Dios, que nos ama, aunque, a veces, esté oculto y silencioso” (p. 4).

c) Gustavo Flores Quelopana, escritor y filósofo autodidacta de fe católica: Vida sin sentido y olvido de Dios de 2012, donde dice que “(e)l hombre de la modernidad tardía es expresión cabal del nihilismo vital, no solo vive sin Dios, sino que vive de espaldas al prójimo y a sí mismo".

d) Intip Megil Guamán, músico y escritor, Illa. El sentido de la existencia desde una perspectiva tawaísta de 2007(?), en el que afirma que “(e)n la actualidad se ven claramente los vacíos espirituales que surgen en la sociedad, carencias y ausencias que las diversas filosofías o religiones son ineficaces de remediar" (p. 18) y para remediar eso propone volver a la religión andina o tawaísmo para llegar a la Illa o iluminación, y, así, aceptar el fundamento par o la dualidad que rige el cielo y la tierra, vivir en la armonía de la crianza mutua, y tratar de recibir el Illa para, a su vez, retransmitirlo, y producir el equilibrio, crear la diversidad y creer en ella (pp. 119-120).

e) Miguel Polo Santillán, filósofo y docente sanmarquino, Indagaciones sobre el sentido de la vida del 2011, donde declara de forma muy realista que “...el problema del sentido de la vida, además de ser un tema filosófico y social, es un tema político. La política debería dar las condiciones sociales, legales y culturales para que las personas construyan formas de vida más excelentes y sientan sus vidas más realizadas, sin convertir a los ciudadanos en peones o engranajes de un sistema irracional" (p. 66).

Y se han publicado artículos como:

el del autor de la presente reseña, filósofo humanista secular: "La vida humana: ¿cuál es su sentido?” de 1999, donde escribí que “(e)ntonces no importa que no haya un sentido predeterminado para nuestras vidas: nosotros mismos podemos crear y recrear premeditadamente o no- nuestras metas y logros -así como frustraciones y fracasos- en este mundo del cual no tenemos ninguna duda de que exista. Nosotros mismos podemos proyectarnos hacia el futuro e ir construyendo nuestros ideales y anhelos, claro está hasta donde las circunstancias naturales y sociales -que otros supeditan a la Providencia, Destino o Suerte- lo permitan" (p. 8).

el de Octavio Obando, filósofo sanmarquino y docente actualmente en el Brasil: "Ética y sentido de la vida" del 2000, donde sostiene que "(e)l sentido de la vida no es puesto por entidad extrahumana alguna (ni tampoco se realiza en el mundo a partir de entidad extrahumana alguna). Tampoco es puesta por nosotros, es, en rigor, lo que el contexto material y cultural nos ofrece en cierto horizonte de posibilidades” (p. 32). 
Sobre el segundo modo, el pesimista, tenemos libros, como el de Stergios Korfiatis, finado filósofo peruano, Solo sé que nada somos de 2007, una autobiografía con reflexiones filosóficas, y el que reseñaremos a continuación de Edgar Gutiérrez Gómez, educador y catedrático universitario, El absurdo de la vida del 2012, un ensayo donde su título ya nos está diciendo cuál es su postura sobre el sentido de la vida o existencia humana. En la introducción empieza hablando del fatalismo del filósofo rumano Emile Ciorán, autor de, entre muchos otros libros Del inconveniente de haber nacido (1973), su siempre reniego del "porqué (sic) vino a este mundo inconsultamente, para luchar contra esta vida llena de problemas" (p. 7). Sí pues, solo los seres humanos tienen otras clases de problemas además del de la supervivencia.

Cosa semejante, dice, sucede "cuando analizamos nuestra existencia absurda del buen vivir, justificando que somos seres sociales por naturaleza, para finalmente ser prisioneros de la sociedad que no nos deja vivir como deseamos en nuestra corta existencia" (ídem). Esto es paradójico: necesitamos de los demás para vivir, pero a la vez los demás nos limitan. Luego menciona a Dios que "odia tanto a los humanos y a toda su creación... sabemos que no desea venir...y el día en que aparezca será un rendir de cuentas y pedidos infinitos del hombre para que cumpla sus deseos..." (p. 10). Un reclamo que se justifica por la existencia de la maldad y el sufrimiento humanos. Y sigue en estilo vallejiano “...Dios...cada vez que el hombre quiere acercarse, se aleja más” (ídem)...” Desde que nacimos estamos abocados a buscar a la divinidad, debería ser que él, Dios, venga a nosotros a encararnos de todo (sic) los pedidos que [le] hicimos, que algunos eran tan absurdos como la vida misma" (p. 11). Tal búsqueda no tendría sentido si Dios, como parece, no existiese y simplemente fuese una quimera humana más, producto de nuestra imaginación, necesidad y temor a la realidad social y natural.

Termina el autor su introducción diciendo "Finalmente, puedo estar orgulloso de lo hecho, pero debería estarlo mucho más de lo que no hice. Ese orgullo está cumplido" (id.). Un reclamo que vale para todos: no hemos hecho todo lo que podíamos hacer, muchas veces por distracciones, vanas, pereza, cinismo, ignorancia o cobardía. Su ensayo propiamente dicho empieza en la p. 13 y termina en la p. 129. Reconoce que la palabra "absurdo" fue usado en especial por los filósofos existencialistas a veces catalogados como irracionalistas (p. 13). A continuación, transcribe 12 conceptos sobre el existencialismo (p. 14-38), observando que casi todos ellos remiten a Kierkegaard, y copia, también innecesariamente, en cuatro págs. (40-43), la biografía de él escrita por Copleston. Simplemente debió hacer una síntesis nuestro doctor en educación.

Considera, con razón, a Camus como el "pensador más prolijo" de la filosofía del absurdo de la vida (p. 45) y transcribe dos breves biografías de él (pp. 45-46 y 4950), evitando así nuevamente sintetizar lo que lee. Después Gutiérrez, recalca lo efímero del placer (erótico) pues luego de éste, "llega al fatalismo y el vacío existencial" (p. 47). En general, todo placer es momentáneo, finito. Y si no se tiene otro tipo de placeres, metas, responsabilidades e ideales se puede caer en tal fatalidad y vaciedez. Menciona a Camus y el único "problema filosófico verdaderamente serio: el suicidio. Juzgar si la vida vale o no la pena de vivirla es responder a la pregunta fundamental de la filosofía" (pp. 49-50). Un tema delicado que, debido a las múltiples autoeliminaciones a lo largo de la historia y por todo el mundo, es evidente que mucha gente llegó a una respuesta negativa pero que, 
felizmente, la mayoría a todo lo contrario: que, a pesar de lo absurdo, contradictorio, doloroso e injusto de la vida, ésta vale la pena vivirse con sus pocos y breves momentos de felicidad y dicha que pueda haber.

Y comenta nuestro autor: "Toda nuestra vida gira en función al juego de la vida con la muerte, cada día es un aspirar más al suicidio perpetuo, hasta que llega el momento indicado, ahora o mañana. Siempre tenemos la esperanza de vivir más tiempo... ¿iserá que esta vida longeva vale la pena ser vivida?, regresionando a la vida infantil que requiere cuidado especial, pero para la muerte" (p. 51). Cada momento de la vida que vivimos, en verdad, es una negación de la muerte, cada disfrute lo es del sufrimiento, cada acción buena es el rechazo del mal. Y, ciertamente, podemos llegar a una senectud tristemente senil, con nuestras capacidades físicas y mentales muy deterioradas para luego morir, cosa que puede darse en cualquier momento de la vida.

Luego dice Gutiérrez: “...venimos a este mundo para morir; mejor pudo haber sido, por ejemplo, no nacer para estar preocupado sobre esta vida y pensar regresar de dónde venimos, ..." (p. 56). Puede ser cierto lo que dice Gutiérrez, pero ya hemos nacido, no podemos regresar a ninguna parte, ya estamos preocupados en una serie de cosas mientras vivimos, y también nos puede angustiar la muerte en menor o mayor grado, encualquier etapa de nuestro existir. Añade el autor: ... "La única esperanza que debemos tener es la muerte no hay otra alternativa; ..." (p. 58). Sin embargo, los seres humanos ante el rechazo de la muerte y de la inexistencia se esperanzan en los diversos constructos religiosos, filosóficos y científicos que ellos mismos crearon y que les distraen antes del inexorable final.

Además, él cuestiona la moralina pacata: “... ¿cuál es el argumento racional para estar cuestionando la vida ajena que no nos compete en absoluto.... La sociedad hipócrita siempre cuestionó los supuestos excesos que provoca el hombre en su vida diaria, habría que buscar un parámetro [de] que es la mejor forma de actuar..." (p. 65). Ese es un tema clásico que ya desde la antigüedad, los filósofos se habían planteado, por ejemplo, el parámetro del griego Aristóteles era la práctica de la virtud para evitar el vicio por carencia o exceso, para los medievales era seguir la voluntad de Dios, interpretada por la Iglesia católica, para el filósofo alemán moderno Kant era el cumplimiento del deber, y para el filósofo inglés contemporáneo Mill era el máximo de felicidad para el mayor número de personas.

Por otro lado, habla Gutiérrez de lo absurdo de las guerras donde los soldados “desperdiciaron su tiempo" (p. 67). Y, claro está, sus vidas por la Patria, según la propaganda a la cual fueron expuestos desde su niñez, y no, como nos muestra la historia, en defensa de intereses más burdos y crudos propios de las minorías gobernantes y ricas. Añade: “(e)xisten verdades de las cuáles todo hombre es conocedor..., conoce que va a morir, pero no se queda perplejo ante esta tamaña verdad" (p. 68). Pero a decir verdad algunos sí se asombran y se preocupan tanto así que niegan su envejecimiento maquillándolo pero que inexorablemente terminará con la muerte. Otros temen la muerte, pero buscan consuelo y refugio en alguna religión o filosofía que les prometa alguna forma de existencia eterna. 
Además, escribe: ... "está demostrada la insatisfacción del hombre en este mundo que con nada se contenta; así tenemos que es una nada dentro de la nada, la cual vino a esta realidad para terminar siendo nada" (p. 72). Pero, otra vez, la realidad es que también hay personas que sí se contentan, que están satisfechas y felices con su vida por más simple y llana que sea. De nuevo afirma: "Único camino (la decisión razonable del absurdo de la vida) tomado hacia la felicidad eterna que es la muerte, que nos espera angustiada e impaciente" (p. 74). Pero con la muerte del cerebro ya no hay conciencia ni sensación, tampoco entonces tristeza o alegría. También da una explicación existencial del suicidio y la recalca: “...tal persona para suicidarse posiblemente encontró sentido al absurdo de su vida y lo termina con la muerte; de lo contrario, no encontró ninguna razón para vivir y se suicida" (pp. 77-8). Para él, entonces tanto encontrar absurda la vida, una forma de hallarle sentido, como no encontrar por qué vivir llevan a la autoeliminación.

Cuestiona nuestro autor la libertad de los humanos al afirmar que somos dependientes de nuestras creencias: "Creen [los hombres] que son libres en absoluto; sin embargo, la creencia se suma y multiplica con la fe en alguien que puede ser objeto, materia, sus padres, los hijos, bienes materiales o Dios todo poderoso; resulta que este hombre nunca vive libre en absoluto, porque está atado a su creencia en alguien a quien considera importante como razón de su existencia y justamente allí plantea que vivirá hasta que Dios disponga, y él, Dios, dispone cuando lo desea; ésta sería la manera de vivir con una dependencia total dejando nuestra existencia real para estar sumidos y atados a situaciones ajenas a nosotros" (pp. 78-9). Es inevitable, por lo tanto, creer en algo o alguien y depender de eso o ese, puesto que nos da un sentido de orientación y uno de devoción o apego.

Y menciona una verdad: somos seres sociales, nos debemos a los demás al decir: "El hombre dejó de vivir para sí mismo desde la concepción misma y al ser parte integrante de las personas que lo rodean, generalmente, pensando lo mejor para él; se ignora que se está haciendo daño al privar su libertad, exigiendo que actúe como lo desea el padre, los abuelos, los tíos, los héroes o simplemente las familias modelo, aparentemente de una moral intachable. Con la [sic] cual siempre los comparan como el "fulanito tal" para ser aceptado dentro del canon social; ..." (p. 83). Cada sociedad tiene su propia moral y así patrón de aceptación o rechazo de sus miembros que, normalmente, nacen dentro de una familia.

Dice otra gran verdad: "Somos unos locos que fingimos ser racionales, ejemplos de moral y de vida, nos falta una seriedad existencial, porque desde que nacimos entramos a una locura total para sobrevivir en este mundillo de nadie y a la vez de todos, supuestamente" (p. 101). En realidad, aunque tenemos un cerebro que nos permite ser racionales, no siempre lo somos, muchas veces estamos a merced de nuestros miedos, prejuicios, pasiones y supersticiones. Los revolucionarios y "otras personas que murieron por alguna causa justa. Los que creían haber realizado algo para terminar en la nada..." (p. 118). Ciertamente, tanto justos como injustos terminaremos muriendo. Agrega Gutiérrez: "(v)ivimos eternamente engañados, argumentando que es realidad, aunque sea pura ilusión al estilo de los idealistas subjetivos; algunos seres humanos nos hemos ilusionado exageradamente y buscamos algo que perdure para siempre; ...” (p. 121). Si, 
pues, aunque la realidad es todo lo que nos rodea, la interpretamos de acuerdo a nuestras preconcepciones de ella, según lo que hemos aprendido desde niños.

Además, escribe que “(e)xisten lugares donde el hombre puede reflexionar de lo absurdo que es la vida como son los cementerios, velorios, hospitales, iglesias, el propio domicilio donde nació el hombre que va a morir, muy pronto, con el promedio de vida que tenemos en el sentido natural de menos de sesenta años de edad, y los goces se terminan para pensar en el tiempo perdido que dejamos atrás" (p. 122). Ante la enfermedad o la muerte, recién podemos ser conscientes de los preciosos, invaluables e irrepetibles momentos no aprovechados. Sigue esa idea al afirmar "...no debemos desaprovechar el placer de la existencia de la vida que nos depara este mundo” (p. 124). Placer único mientras se viva y se sienta en contraposición al dolor y sufrimiento que también forman parte del vivir. Concordamos también con este autor cuando afirma que "(l)as limitaciones económicas solo crean barreras ideales, pues para vivir bien no se necesita dinero solo una buena predisposición de que la vida es una y es tal y como la sentimos..." (ídem). Pero claro, vivimos dentro del marco de una civilización dineraria donde casi todo se vende y compra, y sin plata no hay comida, ropa, medicamentos y vivienda, por hablar de lo más básico.

El colega añade: “...el hombre, lamentablemente no sabe para qué está viviendo o vino a este mundo, para autodestruirse con sus lamentaciones y decisiones absurdas de que la vida tiene sentido" (p. 125). Por supuesto que mientras vivamos son inevitables el dolor y el sufrimiento y no existe EL sentido de la vida. Sin embargo, Gutiérrez afirma: "Estamos inmersos dentro de la vida infeliz por desear algo mejor, siempre queda algo por hacer en este mundo del absurdo vivir; alguien busca vivir por los hijos, por los padres, por sí mismos; finalmente otros sabotean nuestra existencia a modo y medida de nuestra posibilidad, en relación con la naturaleza". Y, evidentemente nuestro autor encontró su sentido de vivir en su hijo cuya foto de bebé adorna la carátula de su libro.

\section{REFERENCIAS BIBLIOGRÁFICAS}

Chávez, Moisés. s/f. Filosofía de la vida. Lima: Editoriales Unidas.

Flores Quelopana, Gustavo. 2012. Vida sin sentido y olvido de Dios. Lima: IIPCIAL.

Korfiatis, Stergios. 2007. Solo sé que nada somos. Lima: Editorial Gutemberg.

Mackay, Juan A. 1978. El sentido de la vida y otros ensayos. Lima: Ediciones Sanadresinas, 3ra. edición.

Obando, Octavio. "Ética y sentido de la vida” en Paz y Miño, M.A. (ed.), 2000.

Paz y Miño, Manuel Abraham. 1999. Ethos: ¡Vivamos mejor! Una introducción a los problemas de la vida. Lima: Ediciones RPFA.

Paz y Miño, M.A. (ed.), Carmen Zavala, Frederick Edwords, Octavio Obando, y Paul Kurtz. 2000. Eupraxophia. Revista Humanista Secular. El sentido la vida. Lima, Año 1, nro. 2, junio.

Peña Benito, Angel. 2006. Vale la pena vivir. Lima: s/i. 
Polo Santillán, Miguel. 2011. Indagaciones sobre el sentido de la vida. Lima: Fondo Editorial de la UIGV.

\section{CITAR COMO:}

Paz y Miño Conde, M. A. (2019). Gutiérrez Gómez, Edgar (2012). El absurdo de la vida. Ayacucho (Perú): copygraph Bautista, 129 pp. Puriq, 1(01), 107-113. https://doi.org/10.37073/puriq.1.01.17 